\title{
SOME EXTREME FORMS DEFINED IN TERMS OF ABELIAN GROUPS
}

\author{
E. S. BARNES and G. E. WALL
}

(rec. 15 Jan. 1959)

\section{Introduction}

Let $f(\boldsymbol{x})=f\left(x_{1}, x_{2}, \ldots, x_{n}\right)$ be a positive definite quadratic form of determinant $D$, and let $M$ be the minimum of $f(\boldsymbol{x})$ for integral $\boldsymbol{x} \neq \mathbf{0}$. Then we set

$$
\gamma_{n}(f)=M / D^{1 / n}
$$

and

$$
\gamma_{n}=\max \gamma_{n}(f)
$$

the maximum being over all positive forms $f$ in $n$ variables. $f$ is said to be extreme if $\gamma_{n}(f)$ is a local maximum for varying $f$, absolutely extreme if $\gamma_{n}(f)$ is an absolute maximum, i.e. if $\gamma_{n}(f)=\gamma_{n}$.

It is well known that $\gamma_{n}$ is of order $n$ for large $n$; in fact, by the classical results of Blichfeldt and Hlawka (see [3], Ch. II, §6),

$$
\frac{1}{2 \pi e} \leqq \lim \inf \frac{\gamma_{n}}{n} \leqq \lim \sup \frac{\gamma_{n}}{n} \leqq \frac{1}{\pi e} \text {. }
$$

On the other hand, no one has yet constructed a sequence of forms for which $\gamma_{n}(f)$ is unbounded, let alone of order $n$, as $n \rightarrow \infty$. We have therefore thought it worthwhile to describe, in some detail, a new class of extreme forms yielding values $\gamma_{n}(f)$ of order $n^{\frac{1}{2}}$ for suitable large $n$.

More specifically, corresponding to each $N=2^{n}(n=2,3, \ldots)$ and to each sequence of integers $\lambda_{0}, \lambda_{1}, \ldots, \lambda_{n}$ satisfying

$$
\lambda_{0}=0, \lambda_{r}-1 \leqq \lambda_{r-1} \leqq \lambda_{r} \quad(1 \leqq r \leqq n),
$$

we construct a positive $N$-variable form $f_{(\lambda)}$, which we show to be extreme in most cases. We prove also that, for each $N$, there is an $f_{(\lambda)}$ satisfying $\gamma_{N}(f)=\left(\frac{1}{2} N\right)^{\frac{1}{2}}$, whence

$$
\gamma_{N} \geqq\left(\frac{1}{2} N\right)^{\frac{1}{2}} \text { for } N=2^{n}, n \geqq 2 .
$$

Our method of construction is based on the structure of the elementary Abelian group of order $2^{n}$. 
Further investigation shows that by an elaboration of the method, or by using a similar method based on Abelian groups of exponent 3, (1.4) can be strengthened for large $N$. It should also be noted that (1.4) is precise for $N=4$ or 8 , since $\gamma_{4}=\sqrt{ } 2, \gamma_{8}=2$, and that, for sufficiently small $N$, (1.4) is a considerable improvement on known results.

The general properties of forms and lattices which we require are collected in $\S 2$. The forms $f_{(\lambda)}$ and their lattices $\Lambda_{(\lambda)}$ are defined in $\S 3$, the determinant $D$ and minimum $M$ are calculated and a criterion for the minimal vectors is given. In $\S 4$, we prove that the $f_{(\lambda)}$ are extreme (under suitable conditions) and investigate the equivalences between them. In $\S 5$, we enumerate the minimal vectors. A table of the inequivalent extreme forms $f_{(\lambda)}$ in 4,8 , 16 and 32 variables is given at the end of the paper.

\section{Forms and lattices*}

A positive quadratic form $f(x)=f\left(x_{1}, x_{2}, \ldots, x_{n}\right)$ is said to have lattice $\Lambda$ if

$$
f(\boldsymbol{x})=\xi^{\prime} \xi
$$

where $\xi$ runs through the points of $\Lambda$ when $\boldsymbol{x}$ runs through all integral vectors; i.e. if

$$
f(\boldsymbol{x})=\boldsymbol{x}^{\prime} A \boldsymbol{x}=\boldsymbol{x}^{\prime} T^{\prime} T \boldsymbol{x}
$$

where $\Lambda$ is specified by

$$
\xi=T \boldsymbol{x}, \boldsymbol{x} \text { integral. }
$$

Then $D(f)=\operatorname{det} A=(\operatorname{det} T)^{2}=d^{2}(\Lambda)$.

Clearly, if $f$ has lattice $A$, it also has lattice $R A$, where $R$ is any orthogonal transformation. Also, equivalent forms correspond to the same lattice; for if $U$ is an integral unimodular transformation, then $T$ and $T U$ define the same lattice and correspond to the equivalent forms $\boldsymbol{x}^{\prime} A \boldsymbol{x}$ and $\boldsymbol{x}^{\prime} U^{\prime} A U \boldsymbol{x}$. It is thus easy to see that two forms $f_{1}, f_{2}$ with lattices $\Lambda_{1}, \Lambda_{2}$ are equivalent if and only if $\Lambda_{2}=R \Lambda_{1}$ for some orthogonal transformation $R$.

We define the reciprocal lattice $\Lambda^{-1}$ of $\Lambda$ to be the set of points $\eta=T^{\prime-1} \boldsymbol{X}$, $\boldsymbol{x}$ integral, where $\Lambda$ is given by $(2.1)$. Then $d\left(\Lambda^{-1}\right) d(\Lambda)=1$, and the corresponding quadratic forms $\boldsymbol{x}^{\prime} T^{\prime} T \boldsymbol{x}$, and $\boldsymbol{x}^{\prime}\left(T^{\prime} T\right)^{-1} \boldsymbol{x}$ are reciprocal (i.e. have inverse matrices).

We shall be concerned in this paper only with forms whose lattices are sublattices of the integer lattice $\Gamma$. For these, it is convenient to define the notion of dual lattices modulo $k$.

Let $k$ be a positive integer, and $\Lambda_{1}, \Lambda_{2}$ lattices such that

$$
k \Gamma \subset \Lambda_{1}, \Lambda_{2} \subset \Gamma \text {. }
$$

* For further general information we refer the reader to Coxeter's paper [2]. 
Then $\Lambda_{2}$ is said to be the dual of $\Lambda_{1}$ modulo $k$ if it consists of those $x \in \Gamma$ satisfying

$$
\boldsymbol{x}^{\prime} \boldsymbol{y} \equiv 0(\bmod k) \text { for all } \boldsymbol{y} \in \Lambda_{1} .
$$

It is easy to see that in fact

$$
\Lambda_{2}=k \Lambda_{1}^{-1} \text {. }
$$

For if $\Lambda_{1}$ is defined by (2.1), i.e. $\Lambda_{1}=T \Gamma$, then an integral $\boldsymbol{x} \in \Lambda_{2}$ if and only if

$$
\boldsymbol{x}^{\prime} T \boldsymbol{y} \equiv 0(\bmod k) \text { for all } \boldsymbol{y} \in \Gamma,
$$

i.e. if and only if

$$
T^{\prime} \boldsymbol{x} \in k \Gamma \text {. }
$$

Now since $k \Gamma \subset A_{1}$, the relation $k \boldsymbol{u}=T \boldsymbol{x}$ has a solution $\boldsymbol{x} \in \Gamma$ for every $\boldsymbol{u} \in \Gamma$, so that $k T^{-1}$ is an integral matrix. Hence (2.4) is equivalent to

$$
\boldsymbol{x} \in k T^{-1} \Gamma=k \Lambda_{1}^{-1}
$$

and $(2.3)$ is established.

From (2.3), we see that the relation between $\Lambda_{1}$ and $\Lambda_{2}$ is symmetrical, so that also $A_{1}$ is the dual of $\Lambda_{2}$ modulo $k$; and

$$
d\left(\Lambda_{1}\right) d\left(\Lambda_{2}\right)=d(k \Gamma)=k^{n}
$$

Further, if $f_{1}, f_{2}$ are the quadratic forms corresponding to the dual lattices $\Lambda_{1}, A_{2}$, then each of $f_{1}, f_{2}$ is a multiple of the reciprocal of the other.

\section{The Form $f_{(\lambda)}$ and its Lattice}

Let $V$ be the $n$-dimensional vector space over the Galois field $G F(2)$; in terms of a basis $\varepsilon_{1}, \ldots, \varepsilon_{n}$, we may write the elements as $\boldsymbol{\alpha}=\sum \alpha_{i} \boldsymbol{\varepsilon}_{\boldsymbol{i}}$ with coordinates $\alpha_{i}$ which are integers taken modulo 2 . The additive group of $V$, which we shall also denote by $V$, is the elementary Abelian group of order $N=2^{n}$. We shall generally use group, rather than vector space, terminology; we shall, however, speak of cosets "of dimension $r$ " or say that a given subgroup "has basis $\alpha_{1}, \alpha_{2}, \ldots$ " Subgroups and cosets of dimension $r$ will be denoted generically by $V_{r}$ and $C_{r}$ respectively.

In $N$-dimensional Euclidean space we consider integral vectors $\boldsymbol{x}=\left(x_{\boldsymbol{\alpha}}\right)$ with coordinates $x_{\boldsymbol{\alpha}}$ indexed by the $N$ elements $\boldsymbol{\alpha}$ of $V$. For symmetry of notation, we write $\boldsymbol{x} \cdot \boldsymbol{y}$ for the scalar product $\boldsymbol{x}^{\prime} \boldsymbol{y}$.

If $W$ is any subset of $V,[W]$ will denote the vector $\boldsymbol{x}$ defined by

$$
x_{\boldsymbol{\alpha}}= \begin{cases}1 & \text { if } \alpha \in W, \\ 0 & \text { if } \boldsymbol{\alpha} \notin W .\end{cases}
$$

Let $\lambda_{0}, \lambda_{1}, \ldots, \lambda_{n}$ be integral exponents satisfying

$$
\lambda_{0}=0, \lambda_{r}-1 \leqq \lambda_{r-1} \leqq \lambda_{r} \text { for } 1 \leqq r \leqq n
$$


We denote by $\Lambda(\lambda)=\Lambda\left(\lambda_{0}, \lambda_{1}, \ldots, \lambda_{n}\right)$ the sublattice of $\Gamma$ generated by all vectors $2^{\lambda_{n-r}\left[C_{r}\right]}$, where $C_{r}$ runs over all cosets in $V$. Clearly

$$
2^{\lambda_{n}} \Gamma \subset \Lambda(\lambda) \subset \Gamma \text {. }
$$

We now define $f_{(\lambda)}$ to be the $N$-dimensional form with lattice $\Lambda(\lambda)$, so that the values assumed by $f_{(\lambda)}$ for integral values of its variables are those of

$$
\boldsymbol{x}^{2}=\sum_{\boldsymbol{\alpha} \epsilon V} x_{\alpha}^{2} \text { for } \boldsymbol{x} \in A(\lambda) .
$$

(We may remark here that the apparently arbitrary restrictions (3.1) involve very little loss of generality. If $\lambda_{0}>0$, we may consider $2^{-\lambda_{0}} \Lambda(\lambda)$, which corresponds to a multiple of $f_{(\lambda)}$. Also if the exponents satisfy only $0 \leqq \lambda_{r} \leqq r(0 \leqq r \leqq n)$, it is not difficult to show that there exists a set $(\lambda)$ defining the same lattice and satisfying (3.1), with the possible exception of the inequality $\lambda_{n}-1 \leqq \lambda_{n-1}$.)

The exponents $\lambda_{r}^{\prime}$ defined by

$$
\lambda_{r}^{\prime}=\lambda_{n}-\lambda_{n-r} \quad(0 \leqq r \leqq n)
$$

are said to be dual to the exponents $\lambda_{r}$. It is evident that $\left(\lambda^{\prime}\right)$ satisfies (3.1), that $\lambda_{n}^{\prime}=\lambda_{n}$, and that $(\lambda)$ is dual to $\left(\lambda^{\prime}\right)$.

We can now prove

THEOREM 3.1. (i) $\Lambda(\lambda)$ and $\Lambda\left(\lambda^{\prime}\right)$ are dual lattices modulo $2^{\lambda_{n}} ; f_{(\lambda)}$ and $f_{(\lambda,)}$ are multiples of reciprocal forms.

(ii) Let $\varepsilon_{1}, \ldots, \varepsilon_{n}$ be any basis of $V$. Then a basis of $\Lambda(\lambda)$ is given by the $N$ vectors

$$
2^{\lambda_{n-r}}\left[V_{r}\right]
$$

where $V_{r}$ runs through the subgroups of $V$ which have a subset of $\varepsilon_{1}, \ldots, \varepsilon_{n}$ as basis.

(iii) The determinants $d(\lambda), D(\lambda)$ of $\Lambda(\lambda), f_{(\lambda)}$ are given by

$$
\begin{aligned}
& \log _{2} d(\lambda)=\sum_{r=0}^{n} \lambda_{r}\left(\begin{array}{l}
n \\
r
\end{array}\right), \\
& \log _{2} D(\lambda)=2 \sum_{r=0}^{n} \lambda_{r}\left(\begin{array}{l}
n \\
r
\end{array}\right) .
\end{aligned}
$$

Proof. (a) We first show that

$$
\boldsymbol{x} \cdot \boldsymbol{y} \equiv 0\left(\bmod 2^{\lambda_{n}}\right) \text { if } \boldsymbol{x} \in \Lambda(\lambda), y \in \Lambda\left(\lambda^{\prime}\right) .
$$

For this, it suffices to prove that for any $\operatorname{cosets} C_{r}, C_{s}^{\prime}$

$$
2^{\lambda_{n-r}+\lambda_{n-s}^{\prime}}\left[C_{r}\right] \cdot\left[C_{s}^{\prime}\right] \equiv 0 \quad\left(\bmod 2^{\lambda_{n}}\right) .
$$

Now if $r+s \leqq n$, then 


$$
\lambda_{n-r}+\lambda_{n-s}^{\prime}=\lambda_{n}+\lambda_{n-r}-\lambda_{s} \geqq \lambda_{n}
$$

and (3.7) is trivial. If $r+s>\dot{n}$, then $C_{r} \cap C_{s}^{\prime}$ is either empty or a coset of dimension at least $r+s-n$; in either case

$$
\left[C_{r}\right] \cdot\left[C_{s}^{\prime}\right] \equiv 0 \quad\left(\bmod 2^{r+s-n}\right) .
$$

Since, by (3.1), $\lambda_{s}-\lambda_{n-r} \leqq s-(n-r)$, we have

$$
\lambda_{n-r}+\lambda_{n-s}^{\prime}+r+s-n=\lambda_{n}+r+s-n-\left(\lambda_{s}-\lambda_{n-r}\right) \geqq \lambda_{n},
$$

and (3.7) follows at once.

(b) With the notation of part (ii) of the theorem, let $\Lambda_{1}(\lambda)$ be the lattice spanned by the $N$ vectors (3.3). Then clearly

$$
2^{\lambda_{n}} \Gamma \subset \Lambda_{1}(\lambda) \subset \Lambda(\lambda) \text {. }
$$

Further, we show that

$$
\log _{2} d\left(\Lambda_{1}(\lambda)\right)=\sum_{r=0}^{n} \lambda_{r}\left(\begin{array}{l}
n \\
r
\end{array}\right)
$$

Since there are $\left(\begin{array}{l}n \\ r\end{array}\right)$ vectors $2^{\lambda_{n-r}}\left[V_{r}\right]$ for each $r,(3.9)$ will follow when we show that the set of all vectors $\left[V_{r}\right]$ forms a basis of $\Gamma$. To see this, suppose the $\left[V_{r}\right]$ ordered in such a way that the dimensions $r$ do not decrease. Then, for each $V_{r}$, there is an $\alpha \in V$ such that $[\boldsymbol{\alpha}]$ has coefficient 1 in $\left[V_{r}\right]$ and coefficient 0 in any predecessor of $\left[V_{r}\right]$; in fact, if $\varepsilon_{i_{1}}, \ldots, \varepsilon_{i_{r}}$ is a basis of $V_{r}, \boldsymbol{\alpha}=\boldsymbol{\varepsilon}_{\boldsymbol{i}_{1}}+\ldots+\boldsymbol{\varepsilon}_{\boldsymbol{i}_{r}}$ satisfies this requirement. Hence the $N$ unit vectors $[\boldsymbol{\alpha}](\boldsymbol{\alpha} \in V)$ are integral linear combinations of the $\left[V_{r}\right]$, whence the $\left[V_{r}\right]$ form a basis of $\Gamma$, as required.

(c) Let $\Lambda_{1}\left(\lambda^{\prime}\right)$ be defined as in (b) for the exponent set $\left(\lambda^{\prime}\right)$. By (3.9), the determinants $d_{1}, d_{1}^{\prime}$ of $\Lambda_{1}(\lambda), \Lambda_{1}\left(\lambda^{\prime}\right)$ satisfy

whence

$$
\begin{aligned}
\log _{2}\left(d_{1} d_{1}^{\prime}\right) & =\sum_{r=0}^{n} \lambda_{r}\left(\begin{array}{l}
n \\
r
\end{array}\right)+\sum_{r=0}^{n} \lambda_{n-r}^{\prime}\left(\begin{array}{c}
n \\
n-r
\end{array}\right) \\
& =\sum \lambda_{n}\left(\begin{array}{l}
n \\
r
\end{array}\right) \\
& =\lambda_{n} 2^{n}=\log _{2}\left(2^{N \lambda_{n}}\right)
\end{aligned}
$$

$$
d_{1} d_{1}^{\prime}=d\left(2^{\lambda_{n}} \Gamma\right)
$$

From this, and (3.6), it follows that $\Lambda_{1}(\lambda)$ and $A_{1}\left(\lambda^{\prime}\right)$ are dual lattices modulo $2^{\lambda_{n}}$. But it also follows from (3.6) that $\Lambda(\lambda)$ is contained in the dual of $\Lambda_{1}\left(\lambda^{\prime}\right)$, i.e. that $A(\lambda) \subset \Lambda_{1}(\lambda)$. It follows from (3.8) that therefore

$$
\Lambda_{1}(\lambda)=\Lambda(\lambda) \text {. }
$$

All parts of the theorem now follow at once after identifying $\Lambda(\lambda), A\left(\lambda^{\prime}\right)$ with the dual lattices $\Lambda_{1}(\lambda), \Lambda_{1}\left(\lambda^{\prime}\right)$. 
As a corollary, we obtain

LEMMA 3.1. $\Lambda(\lambda)$ is the set of integral $\boldsymbol{x}$ satisfying the system of congruences

$$
\sum_{\boldsymbol{\alpha} \in C_{r}} x_{\boldsymbol{\alpha}} \equiv 0 \quad\left(\bmod 2^{\lambda_{r}}\right)
$$

taken over all cosets $C_{r}$ of $V$.

Proof. By Theorem 2.1, $\Lambda(\lambda)$ is the dual, modulo $2^{\lambda_{n}}$, of $\Lambda\left(\lambda^{\prime}\right)$, which is generated by the vectors $2^{\lambda^{\prime}{ }_{n-r}}\left[C_{r}\right]$. Hence, from the definition of dual lattices, $x \in \Lambda(\lambda)$ if and only if $x \in \Gamma$ and

$$
2^{\lambda_{n-r}}\left[C_{r}\right] \cdot x \equiv 0\left(\bmod 2^{\lambda_{n}}\right) \quad(0 \leqq r \leqq n) .
$$

Since $\lambda_{n-r}^{\prime}=\lambda_{n}-\lambda_{r}$, these are precisely the congruences (3.10).

LEMma 3.2. $\Lambda(\lambda)$ is invariant under the following orthogonal transformations:

(i) the permutation of the coordinates $x_{\boldsymbol{\alpha}}$ induced by the transformation

$$
\alpha \rightarrow \tau \alpha+\gamma
$$

of $V$, where $\tau$ is a non-singular matrix over $G F(2)$ and $\gamma$ is any fixed element of $V$;

(ii) the involution

$$
y_{\boldsymbol{\alpha}}=\left\{\begin{aligned}
x_{\boldsymbol{\alpha}} & \text { if } \boldsymbol{\alpha} \in W, \\
-x_{\boldsymbol{\alpha}} & \text { if } \boldsymbol{\alpha} \notin W,
\end{aligned}\right.
$$

where $W$ is any fixed subgroup of $V$ of dimension $n-1$.

Proof. (i) The transformation (3.11) of $V$ permutes the cosets of each dimension $r$, and so induces a permutation of the generators $2^{\lambda_{n-r}}\left[C_{r}\right]$ of $\Lambda(\lambda)$.

(ii) Suppose first that $\boldsymbol{x}=2^{\lambda_{n-r}}\left[C_{r}\right]$ for some coset $C_{r}$. Then $C_{r} \cap W$ is either empty or a coset of dimension at least $r-1$, and in each case it is easy to show that $y$, defined by (3.12), is a point of $\Lambda(\lambda)$. For if $C_{r} \cap W$ is empty, $\boldsymbol{y}=-\boldsymbol{x}$; if $C_{r} \cap W=C_{r}, \boldsymbol{y}=\boldsymbol{x}$; and if $C_{r} \cap W=C_{r-1}$ and say $C_{r}=C_{r-1} \cup C_{r-1}^{\prime}$,

$$
\begin{aligned}
y & =2^{\lambda_{n-r}}\left[C_{r-1}\right]-2^{\lambda_{n-r}}\left[C_{r-1}^{\prime}\right] \\
& =2^{\lambda_{n-r}+1}\left[C_{r-1}\right]-2^{\lambda_{n-r}}\left[C_{r}\right] \\
& \epsilon \Lambda(\lambda)
\end{aligned}
$$

since $\lambda_{n-r}+1 \geqq \lambda_{n-r+1}$.

Since $A(\lambda)$ is generated by the vectors $2^{\lambda_{n-r}}\left[C_{r}\right]$, it follows that $y \in A(\lambda)$ whenever $x \in \Lambda(\lambda)$. Since the transformation (3.12) is involutory, the converse statement holds. $\Lambda(\lambda)$ is therefore invariant, as asserted.

Our final task in this section is to determine the minimum $M$ of $f_{(\lambda)}$, i.e. the minimum of $\boldsymbol{x}^{2}$ for points $\boldsymbol{x} \neq \mathbf{0}$ of $\Lambda(\lambda)$. We shall show that in fact 
$M$ is the minimum of $\boldsymbol{x}^{2}$ over the set of vectors $2^{\lambda_{n-r}}\left[C_{r}\right]$ which we have selected to generate $\Lambda(\lambda)$.

For this purpose, it is convenient to define the special lattices $\Lambda_{s}(0 \leqq s \leqq n): \Lambda_{s}$ is the lattice $\Lambda(\lambda)$ whose exponents are defined by

$$
\lambda_{r}=0 \text { if } r \leqq s ; \lambda_{r}=1 \text { if } r>s .
$$

Lemma 3.3. Suppose that $\boldsymbol{x} \in \Lambda_{s}$ and that not all $x_{\boldsymbol{\alpha}}$ are even. Then at least $2^{n-s}$ coordinates $x_{\alpha}$ are odd.

Proof. If $s=n$, the result is trivial; hence we may suppose that $0 \leqq s<n$. We shall proceed by induction on $n$, the result being obvious when $n=1$.

After applying a suitable transformation (3.11), we may suppose that $x_{0}$ is odd. By (3.10), with $r=n, \lambda_{n}=1$, we have

$$
\sum_{\boldsymbol{\alpha} \in V} x_{\boldsymbol{\alpha}} \equiv 0(\bmod 2) \text {, }
$$

so that some other coordinate $x_{\gamma}$, say, is odd. Choose an $(n-1)$-dimensional subgroup $W$ of $V$ so that $\gamma \notin W$, and let $W^{\prime}$ be the other coset of $W$.

Now since (3.10) holds a fortiori whenever $C_{r} \subset W$, induction on $n$ shows that $x_{\alpha}$ is odd for at least $2^{(n-1)-s}$ indices $\boldsymbol{\alpha}$ in $W$. The same result also holds for $W^{\prime}$; for, by the transformation $\boldsymbol{\alpha} \rightarrow \boldsymbol{\alpha}+\boldsymbol{\gamma}$ of (3.11), $W^{\prime}$ is transformed into $W$; and, under the induced permutation of the coordinates, $x_{\gamma}$, which is odd, is transformed into $x_{\mathbf{0}}$. Thus the odd coordinates $x_{\boldsymbol{\alpha}}$ number at least $2^{n-1-s}+2^{n-1-s}=2^{n-s}$, as asserted.

Let us now define the rank of a point $\boldsymbol{x} \neq \mathbf{0}$ of $\Lambda(\lambda)$ to be the largest $r(0 \leqq r \leqq n)$ for which all coordinates $x_{\alpha}$ are divisible by $2^{\lambda_{r}}$. We then have

THEOREM 3.2. The minimum $M$ of $f_{(\lambda)}$ is given by

$$
\log _{2} M=m=\min _{r}\left(n-r+2 \lambda_{r}\right) .
$$

$A$ point $\boldsymbol{x} \neq \mathbf{0}$ of $\Lambda(\lambda)$ is a minimal vector $f_{(\lambda)}$ if and only if it is of rank $R$, where

$$
n-R+2 \lambda_{R}=m,
$$

and, for some subset $H$ of $V$ containing $2^{n-n}$ elements,

$$
\left|x_{\boldsymbol{\alpha}}\right|=2^{\lambda} \text { if } \boldsymbol{\alpha} \epsilon H, x_{\boldsymbol{\alpha}}=0 \text { if } \boldsymbol{\alpha} \notin H .
$$

Proof. Each generator $\boldsymbol{x}=2^{\lambda_{r}}\left[C_{n-r}\right]$ of $\Lambda(\lambda)$ satisfies

$$
\boldsymbol{X}^{2}=2^{n-r+2 \lambda_{r}},
$$

so that certainly $M \leqq 2^{m}$, where $m$ is defined by (3.14).

On the other hand, let $\boldsymbol{x} \in \Lambda(\lambda), \boldsymbol{x} \neq \mathbf{0}$, and suppose that $\boldsymbol{x}$ has rank $r(0 \leqq r \leqq n)$; set $\boldsymbol{y}=2^{-\lambda_{r}} \boldsymbol{X}$, so that $\boldsymbol{y}$ is integral, $\boldsymbol{y} \neq \mathbf{0}$.

If now all $y_{\boldsymbol{\alpha}}$ are even, then, by the definition of rank, we must have 
$r=n$; since $\boldsymbol{y} \neq \mathbf{0}$, we therefore have

$$
x^{2}=2^{2 \lambda_{n}} y^{2} \geqq 4 \cdot 2^{2 \lambda_{n}}>2^{m} .
$$

If however some $y_{\boldsymbol{\alpha}}$ is odd, we see that $y \in A_{\boldsymbol{r}}$. For, since

$$
\boldsymbol{x} \in \Lambda\left(\lambda_{0}, \lambda_{1}, \ldots, \lambda_{n}\right), y=2^{-\lambda_{r}} \boldsymbol{X} \in \Lambda\left(0, \ldots, 0, \lambda_{r+1}-\lambda_{r}, \ldots, \lambda_{n}-\lambda_{r}\right) ;
$$

we have $\lambda_{r+1}>\lambda_{r}$, by the definition of rank, and so $\lambda_{s}-\lambda_{r} \geqq 1$ for $s>r$; hence a fortiori $y \in \Lambda(0, \ldots, 0,1, \ldots, 1)=\Lambda_{r}$. Now Lemma 3.3 shows that at least $2^{n-r}$ coordinates $y_{\boldsymbol{\alpha}}$ are odd, whence

$$
\boldsymbol{x}^{2}=2^{2 \lambda_{r}} \boldsymbol{y}^{2} \geqq 2^{n-r+2 \lambda_{r}} \geqq 2^{m} .
$$

This establishes (3.14). The argument shows that in fact $x^{2}=M=2^{m}$ precisely when $\boldsymbol{x}$ has rank $R$ satisfying (3.15) and the corresponding $\boldsymbol{y}=2^{-\lambda_{R}} \boldsymbol{X}$ has $2^{n-R}$ coordinates \pm 1 and the rest zero. The proof of the theorem is therefore complete.

\section{The Extreme Forms $f_{(\lambda)}$}

Although Theorem 3.2 takes us some way towards a specification of the minimal vectors of $f_{(\lambda)}$, the complete picture is rather complicated. (We shall give more precise results in $\S 5$.) However, we can easily write down a sufficiently large set of minimal vectors to enable us to establish the extreme forms $f_{(\lambda)}$.

We denote generically by $R$ an index satisfying (3.15), so that there are certainly minimal vectors of rank $R$.

Let $\mathfrak{M}_{R}$ denote the set of vectors

$$
2^{\lambda}\left[C_{n-R}\right], \quad 2^{\lambda}\left[C_{n-R-1}\right]-2^{\lambda_{R}}\left[C_{n-R-1}^{\prime}\right]
$$

and their negatives, taken over all cosets of the indicated dimensions, where $C_{n-R-1}, C_{n-R-1}^{\prime}$ denote distinct cosets of the same subgroup.

LEMma 4.1. (i) $\mathfrak{M}_{R}$ is a set of $2^{n+1} K_{n, R}$ minimal vectors of $f_{(\lambda)}$ of rank $R$, where

$$
K_{n, R}=\frac{\left(2^{n}-1\right)\left(2^{n-1}-1\right) \ldots\left(2^{n-R+1}-1\right)}{\left(2^{R}-1\right)\left(2^{R-1}-1\right) \ldots(2-1)} .
$$

(ii) The group (GS of automorphs of $f_{(\lambda)}$ is transitive on $\mathfrak{M}_{R}$.

Proof. Let ()$^{\prime}$ ' be the group generated by all the orthogonal transformations given in Lemma 3.2. Since these leave $A(\lambda)$ invariant, (15' is a subgroup of (S).

It is now easy to see that $\mathfrak{M}_{R}$ is precisely the set of vectors which are the transforms by 16 ' of any one of them. For, by suitable choice of $\tau$ and $\gamma$ in (3.11), any coset may be mapped into any other coset of the same dimension; and, for fixed $C_{n-R-1}, C_{n-R-1}^{\prime}$ with $C_{n-R-1} \cup C_{n-R-1}^{\prime}=C_{n-R}$, 
the transformation (3.12) interchanges the two vectors (4.1) if $W$ is suitably chosen.

Since $2^{\lambda} R\left[C_{n-R}\right]$ is a generator of $\Lambda(\lambda)$, all vectors of $\mathfrak{M}_{R}$ belong to $\Lambda(\lambda)$; and, by the criterion of Theorem 3.2, they are all minimal vectors of $f_{(\lambda)}$. The argument also establishes part (ii) of the lemma.

Finally, if $V_{n-R}$ is any fixed subgroup, we have from (4.1) the 2 vectors $\pm 2^{\lambda_{R}\left[V_{n-R}\right]}$ and the $2\left(2^{n-R}-1\right)$ vectors $2^{\lambda_{R}}\left[C_{n-R-1}\right]-2^{\lambda_{R}}\left[C_{n-R-1}^{\prime}\right]$ obtained by splitting $V_{n-R}$ in all ways into 2 cosets. This gives $2^{n-R+1}$ vectors of $\mathfrak{M}_{R}$ corresponding to each $V_{n-R}$. Since $V_{n-R}$ has $2^{R}$ distinct cosets, and $V$ contains $K_{n, R}$ subgroups $V_{n-R}$, the total number of vectors in $\mathfrak{M}_{R}$ is $2^{n+1} K_{n, R}$, as asserted.

Lemma 4.2. If $0<R<n, f_{(\lambda)}$ is perfect with respect to the set $\mathfrak{M}_{R}$ of minimal vectors; i.e. if $g(\boldsymbol{x})$ is any quadratic form satisfying

$$
g(\boldsymbol{x})=0 \text { for all } \boldsymbol{x} \in \mathfrak{M}_{R},
$$

then $g(\boldsymbol{x}) \equiv 0$.

Proof. Suppose that (4.2) holds, where $g(x)=\sum_{\alpha, \beta \epsilon V} b_{\alpha \beta} x_{\alpha} x_{\beta}\left(b_{\alpha \beta}=b_{\beta \alpha}\right)$; we have to show that $b_{\alpha \boldsymbol{\beta}}=0$ for all $\boldsymbol{\alpha}, \boldsymbol{\beta} \in V$.

Inserting the vectors (4.1), with $C_{n-R}=C_{n-R-1} \cup C_{n-R-1}^{\prime}$, we obtain

$$
\begin{gathered}
\sum_{\boldsymbol{\alpha}, \boldsymbol{\beta} \epsilon C_{n-R-1}} b_{\boldsymbol{\alpha} \beta}+b_{\boldsymbol{\alpha}, \boldsymbol{\beta} \in C_{n-R}} b_{\boldsymbol{\alpha} \boldsymbol{\beta}}=0, \\
\sum_{C^{\prime} n-R-1} b_{\boldsymbol{\alpha} \beta}-2 \sum_{\substack{\boldsymbol{\alpha} \in C_{n-R-1} \\
\boldsymbol{\beta} \in C^{\prime} n-R-1}} b_{\boldsymbol{\alpha} \beta}=0,
\end{gathered}
$$

whence, by addition,

$$
\sum_{\boldsymbol{\alpha}, \boldsymbol{\beta} \in C_{n-R-1}} b_{\boldsymbol{\alpha} \beta}+\sum_{\boldsymbol{\alpha}, \boldsymbol{\beta} \in C^{\prime} n-R-1} b_{\alpha \beta}=0 .
$$

Applying (4.4) to each pair of 3 distinct cosets of a $V_{n-R-1}$ (which is possible since $n-R-1 \leqq n-2)$, we deduce that

$$
\sum_{\alpha, \beta \in C_{n-R-1}} b_{\alpha \beta}=0 .
$$

We now make the inductive assumption that the relations

$$
\begin{aligned}
\sum_{\boldsymbol{\alpha} \in C_{\boldsymbol{r}+1}} b_{\boldsymbol{\alpha} \boldsymbol{\beta}} & =0, \\
\sum_{\boldsymbol{\beta} \in C_{\boldsymbol{r}}} b_{\boldsymbol{\alpha} \boldsymbol{\beta}} & =0
\end{aligned}
$$

hold, for some $r$ with $0<r \leqq n-2$, for any cosets $C_{r+1}, C_{r}$, and prove that (4.6) holds for cosets of dimension $r-1$.

Let $V_{r-1}$ be a subgroup and $C_{r-1}=V_{r-1}+\gamma_{1}$ any coset of it. Since $r-1 \leqq n-3, V_{r-1}$ has at least $2^{3}$ cosets; let $C^{i}=V_{r+1}+\gamma_{i}(i=1, \ldots, 6)$ 
be 6 distinct cosets of $V_{r-1}$ such that $C^{i} \cup C^{i+1}(i=1,3,5)$ are 3 cosets of a subgroup $V_{r}$. We write (temporarily)

$$
B_{i j}=\sum_{\substack{\boldsymbol{\alpha} \in C^{i} \\ \boldsymbol{\beta} \in C^{j}}} b_{\boldsymbol{\alpha} \boldsymbol{\beta}}
$$

From (4.6), with $C_{\mathbf{r}}=C^{i} \cup C^{j}$, we have

$$
B_{i i}+B_{j j}+2 B_{i j}=0 \quad(1 \leqq i<j \leqq 6) .
$$

From (4.5), with $C_{r+1}=\cup_{i=1}^{4} C^{i}$, we have

$$
\sum_{i=1}^{4} B_{i i}+\underset{1 \leqq i<j \leqq 4}{2 \sum_{i j}} B_{i j}=0 .
$$

Adding (4.7) for all $i, j$ with $1 \leqq i<j \leqq 4$ and subtracting (4.8), we obtain

$$
\sum_{i=1}^{4} B_{i i}=0 \text {. }
$$

Adding (4.7) for $i, j=1,2$ and $i, j=3,4$, and subtracting (4.9), we obtain

$$
B_{12}+B_{34}=0 \text {. }
$$

From this, and the two similar relations $B_{12}+B_{56}=0, B_{34}+B_{56}=0$, we deduce that $B_{12}=0$; hence, by (4.7),

$$
B_{11}+B_{22}=0 \text {. }
$$

From this and the two similar relations $B_{11}+B_{33}=0, B_{22}+B_{33}=0$, we deduce that $B_{11}=0$, i.e.

$$
\sum_{\boldsymbol{\alpha}, \boldsymbol{\beta} \in C_{r-1}} b_{\boldsymbol{\alpha} \boldsymbol{\beta}}=0
$$

as required.

Now we have shown that (4.5), (4.6) hold for $r=n-R-1$, where $0 \leqq n-R-1 \leqq n-2$. Hence by induction, (4.5) and (4.6) hold for $r=0$. Thus, for any distinct $\alpha, \beta$,

$$
b_{\alpha \boldsymbol{\alpha}}+b_{\beta \beta}+2 b_{\alpha \beta}=0, b_{\alpha \boldsymbol{\alpha}}=0 .
$$

It follows that $b_{\alpha \boldsymbol{\beta}}=0$ for all $\boldsymbol{\alpha}, \boldsymbol{\beta}$, and our proof is complete.

It is now easy to prove our main result:

THEOREM 4.1. $f_{(\lambda)}$ is extreme if and only if it has minimal vectors of rank $R$ for some $R$ with $0<R<n$, or is the 4-variable form $f_{(0,1,1)}$.

Proof. (i) If $R$ satisfies (3.15), with $0<R<n$, we have exhibited a set $\mathfrak{M}_{R}$ of minimal vectors such that (a) the group (S) of automorphs of $f_{(\lambda)}$ is transitive on $\mathfrak{M}_{R}$ (Lemma 4.1); and (b) $f_{(\lambda)}$ is perfect with respect to $\mathfrak{M}_{R}$. Hence, by $[1]$, theorem $4, f_{(\lambda)}$ is extreme.

(ii) If (3.15) holds only with $R=0$ or $R=n$, it may be shown that, when $f_{(\lambda)} \neq f_{(0,1,1)}$, the total number of minimal vectors of rank 0 or $n$ is 
$2^{n+1}=2^{n+1} K_{n, 0}$. Thus $f_{(\lambda)}$ has at most $2^{n+1}=2 N$ pairs of minimal vectors. Since $2 N<\frac{1}{2} N(N+1)$ if $N \geqq 4, f_{(\lambda)}$ is not perfect, and so not extreme, if $N \geqq 4$; and trivially $f_{(\lambda)}$ is not perfect if $N=2$.

Although the $2^{n}$ choices of $(\lambda)$ satisfying (3.1) yield $2^{n}$ distinct forms $f_{(\lambda)}$ for each $n$, most of which are extreme, these forms are not all inequivalent. The following two theorems appear to settle the problem of finding the inequivalent $f_{(\lambda)}$, at least for small $N$.

THEOREM 4.2. For any set $(\lambda)$ of exponents satisfying (3.1), define the conjugate set $(\mu)$ by

$$
\mu_{r}=r+\lambda_{n \rightarrow r}-\lambda_{n}
$$

Then $(\mu)$ satisfies $(3.1)$ and

$$
2^{-\mu_{n}} f_{(\mu)} \sim 2^{-\lambda_{n}} f_{(\lambda)}
$$

Proof. From (4.10),

$$
\mu_{0}=0, \mu_{r}-\mu_{r-1}=1-\left(\lambda_{n-r+1}-\lambda_{n-r}\right)=0 \text { or } 1 \quad(1 \leqq r \leqq n)
$$

so that $(\mu)$ satisfies (3.1). It is also clear that $(\lambda)$ is conjugate to $(\mu)$, i.e. $\lambda_{r}=r+\mu_{n-r}-\mu_{n}$.

Now let $B: \varepsilon_{1}, \varepsilon_{2}, \ldots, \varepsilon_{n}$ be any fixed basis of $V$, and define a scalar product on $V$ by

$$
\alpha \cdot \beta=\sum_{i=1}^{n} \alpha_{i} \beta_{i} \text { if } \alpha=\sum \alpha_{i} \varepsilon_{i}, \beta=\sum \beta_{i} \varepsilon_{i} .
$$

$\boldsymbol{\alpha} \cdot \boldsymbol{\beta}$ is thus an element of $G F(2)$, written as an integer modulo 2 . We now consider the transformation

$$
y_{\boldsymbol{\alpha}}=2^{-\lambda_{n}} \sum_{\boldsymbol{\beta} \in V}(-1)^{\boldsymbol{\alpha} \cdot \boldsymbol{\beta}} x_{\boldsymbol{\beta}},
$$

and we shall show that

$$
\begin{gathered}
y \in \Lambda(\mu) \text { if } x \in \Lambda(\lambda), \\
2^{-\mu_{n}} y^{2}=2^{-\lambda_{n}} x^{2} .
\end{gathered}
$$

By Theorem 3.1. (ii), a basis of $\Lambda(\lambda)$ is given by the vectors $2^{\lambda_{n-r}}\left[V_{r}\right]$, where $V_{r}$ runs through the subgroups of $V$ having a subset of $B$ as basis. Hence, to prove (4.13), it suffices to show that $y \in \Lambda(\mu)$ if $x=2^{\lambda_{n-r}\left[V_{r}\right]}$ $(0 \leqq r \leqq n)$. For each such $V_{r}$, let $V_{n-r}^{\prime}$ be the complementary subgroup of $V$; i.e. $V_{r}$ and $V_{n-r}^{\prime}$ have complementary subsets of $B$ as basis. We then have

$$
\sum_{\beta \in V_{r}}(-1)^{\alpha \cdot \beta}=\left\{\begin{array}{lll}
2^{r} & \text { if } \alpha \in V_{n-r}^{\prime}, \\
0 & \text { if } \alpha \notin V_{n-r}^{\prime}
\end{array}\right.
$$

For if $\alpha \in V_{n-r}^{\prime}$, then $\alpha \cdot \beta=0$ for all $\beta \in V_{r}$. If, however, $\alpha \notin V_{n-r}^{\prime}$, then there is some $\gamma \in V_{r}$ with $\alpha \cdot \gamma=1$; then 


$$
\sum_{\beta \in V_{r}}(-1)^{\alpha \cdot \beta}=\sum_{\beta \in V_{r}}(-1)^{\alpha \cdot(\beta+\gamma)}=-\sum_{\beta \in V_{r}}(-1)^{\alpha \cdot \beta}=0 .
$$

If now $x=2^{\lambda_{n-r}}\left[V_{r}\right],(4.12)$ and (4.15) give

$$
y_{\alpha}=2^{-\lambda_{n}} \sum_{\beta \in V_{r}}(-1)^{\alpha \cdot \beta} 2^{\lambda_{n-r}}= \begin{cases}2^{r+\lambda_{n-r}-\lambda_{n}} & \text { if } \alpha \in V_{n-r}^{\prime}, \\ 0 & \text { if } \alpha \notin V_{n-r}^{\prime},\end{cases}
$$

i.e. $y=2^{\mu_{r}}\left[V_{n-r}^{\prime}\right]$, which is a generator of $\Lambda(\mu)$. This proves (4.13).

To prove $(\mathbf{4 . 1 4})$, we use the case $r=n, V_{r}=V$ of (4.15) to obtain

$$
y^{2}=\sum_{\alpha \in V} y_{\alpha}^{2}=2^{-2 \lambda_{n}} \sum_{\alpha, \beta, \gamma \in V}(-1)^{\alpha-(\beta \overline{+\gamma})} x_{\beta} x_{\gamma}=2^{n-2 \lambda_{n}} \sum_{\alpha \in V} x_{\alpha}^{2},
$$

whence (4.14) follows at once.

The desired equivalence (4.11) follows from (4.13), (4.14), on observing that, since the relations between $(\lambda),(\mu)$ and between $\boldsymbol{x}, \boldsymbol{y}$ are symmetrical, $y \in \Lambda(\mu)$ if and only if $x \in A(\lambda)$.

THEOREM 4.3. For the exponent sets $(\lambda),(\mu)$ given by

$$
\begin{array}{ll}
\lambda_{r}=\left[\frac{r}{2}\right] & (0 \leqq r \leqq n), \\
\mu_{r}=\left[\frac{r+1}{2}\right] & (0 \leqq r \leqq n),
\end{array}
$$

we have

$$
f_{(\mu)} \sim 2 f_{(\lambda)}
$$

Proof. Take any fixed subgroup $W$ of dimension $n-1$ and any element $\gamma \notin W$, and consider the transformation defined by

$$
\begin{aligned}
y_{\alpha} & =x_{\alpha}+x_{\alpha+\gamma} \\
y_{\alpha+\gamma} & =x_{\alpha}-x_{\alpha+\gamma}
\end{aligned} \quad(\alpha \in W)
$$

Then clearly $\boldsymbol{y}^{2}=2 \boldsymbol{x}^{2}$, and so the required equivalence will follow when we show that $y \in \Lambda(\mu)$ if and only if $x \in \Lambda(\lambda)$.

Let $V_{r}$ be any subgroup of $V$ and $\boldsymbol{x}=2^{\lambda_{n-r}}\left[V_{r}\right] \in \Lambda(\lambda)$. Then $V_{r} \cap W$ is either $V_{r}$ or a subgroup $V_{r-1}$; we must now distinguish three cases.

(a) If $V_{r} \subset W,(4.18)$ shows that $y=2^{\lambda_{n-r}}\left[V_{r}\right]+2^{\lambda_{n-r}}\left[V_{r}+\gamma\right]$, i.e. $\boldsymbol{y}=2^{\lambda_{n-r}}\left[V_{r+1}\right]=2^{\mu_{n-r-1}}\left[V_{r+1}\right] \epsilon \Lambda(\mu)$.

(b) If $V_{r} \cap W=V_{r-1}$ and $V_{r}=V_{r-1} \cup\left(V_{r-1}+\gamma\right)$, (4.18) gives

$$
y=2.2^{\lambda_{n-r}}\left[V_{r-1}\right]=2^{\mu_{n-r+1}}\left[V_{r-1}\right] \epsilon \Lambda(\mu) .
$$

(c) The remaining possibility is that $V_{r}=V_{r-1} \cup\left(V_{r-1}+\beta\right)$, where $V_{r-1} \subset W, \quad V_{r-1}+\beta \subset W+\gamma$, but the cosets $V_{r-1}+\beta, V_{r-1}+\gamma$ are distinct. Then observing that $V_{r-1}+\beta+\gamma \subset W$, we obtain from (4.18)

$$
y=2^{\lambda_{n-r}}\left\{\left[V_{r-1}\right]+\left[V_{r-1}+\gamma\right]+\left[V_{r-1}+\beta+\gamma\right]-\left[V_{r-1}+\beta\right]\right\} .
$$


With $V_{r+1}=V_{r-1} \cup\left(V_{r-1}+\beta\right) \cup\left(V_{r-1}+\gamma\right) \cup\left(V_{r-1}+\beta+\gamma\right)$, this gives

$$
\begin{aligned}
y & =2^{\lambda_{n-r}}\left[V_{r+1}\right]-2.2^{\lambda_{n-r}\left[V_{r-1}+\beta\right]} \\
& =2^{\mu_{n-r-1}}\left[V_{r+1}\right]-2^{\mu_{n-r+1}}\left[V_{r-1}+\beta\right] \epsilon \Lambda(\mu) .
\end{aligned}
$$

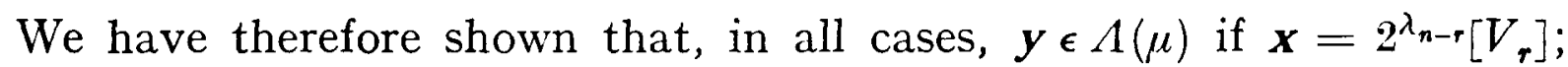
since these vectors generate $\Lambda(\lambda)$, it follows that $y \in A(\mu)$ if $\boldsymbol{x} \in \Lambda(\lambda)$. A precisely similar argument, using the inverse transformation $x_{\alpha}=\frac{1}{2}\left(y_{\alpha}+y_{\alpha+\gamma}\right)$ $x_{\alpha+\gamma}=\frac{1}{2}\left(y_{\alpha}-y_{\alpha+\gamma}\right)$, shows that conversely $\boldsymbol{x} \in \Lambda(\lambda)$ if $\boldsymbol{y} \in \Lambda(\mu)$. This completes the proof of the theorem.

The particular interest of the (equivalent) exponent sets (4.16) and (4.17) is shown by:

THEOREM 4.4. For each $N=2^{n}(n \geqq 2)$, the extreme form $f_{N}$ whose exponents are given by (4.16) has

$$
\gamma_{N}\left(f_{N}\right)=\left(\frac{1}{2} N\right)^{\frac{1}{2}}
$$

and this is the largest value of $\gamma_{N}\left(f_{(\lambda)}\right)$.

Proof. Since $\gamma_{N}(f)=M / D^{1 / N}$, the values of $M$ and $D$ given in Theorems 3.1 and 3.2 show that

$$
\begin{aligned}
\log _{2} \gamma_{N}\left(f_{(\lambda)}\right) & =m-\frac{2}{N} \sum_{r=0}^{n} \lambda_{r}\left(\begin{array}{l}
n \\
r
\end{array}\right) \\
& =\frac{1}{2} n-\frac{1}{N} \sum_{r=0}^{n}\left(n-r+2 \lambda_{r}-m\right)\left(\begin{array}{l}
n \\
r
\end{array}\right) .
\end{aligned}
$$

Since $m=\min \left(n-r+2 \lambda_{r}\right)$, we have $n-r+2 \lambda_{r}-m \geqq 0$ for all $r$; and the parity of $n-r+2 \lambda_{r}-m$ is determined by the parity of $r$. It is thus easy to see that $\gamma_{N}\left(f_{(\lambda)}\right)$ is greatest when the expressions $n-r+2 \lambda_{r}-m$ $(r=0,1, \ldots, n)$ take alternately the values 0 and 1 ; and the only exponent sets satisfying this condition are those given in (4.16) and (4.17). This shows that $\gamma_{N}\left(f_{N}\right)$ is maximal, and a simple calculation now gives (4.19).

It is perhaps worth noting here that, by Theorem 3.1 (i), the reciprocal of $f_{(\lambda)}$ is a multiple of $f_{\left(\lambda^{\prime}\right)}$, where $\lambda_{r}^{\prime}=\lambda_{n}-\lambda_{n-r}(0 \leqq r \leqq n)$. For the exponent set (4.16) corresponding to $f_{N}$, it is easily verified that the dual set $\left(\lambda^{\prime}\right)$ is either (4.16) or (4.17), according as $n$ is odd or even; thus $f_{N}$ is equivalent to (a multiple of) its reciprocal.

\section{The Minimal Vectors of $f_{(\lambda)}$}

For each $R$ satisfying (3.15), we have exhibited a set $\mathfrak{M}_{R}$ of $2^{n+1} K_{n, R}$ minimal vectors of $f_{(\lambda)}$. Denoting by $s_{R}$ the total number of pairs of minimal vectors of rank $R$, we therefore have certainly

$$
2 s_{R} \geqq 2^{n+1} K_{n . R} \text {. }
$$


It is not difficult to obtain an upper bound for $s_{R}$, in the following way. First, the second part of theorem 3.2 may be sharpened to the statement that $\boldsymbol{x} \in \Lambda(\lambda)$ is a minimal vector of rank $R$ if and only if, for some $\operatorname{coset} C_{n-R}$, we have

$$
\left|x_{\boldsymbol{\alpha}}\right|=2^{\lambda_{R}} \text { if } \boldsymbol{\alpha} \epsilon C_{n-R}, x_{\boldsymbol{\alpha}}=0 \text { otherwise. }
$$

We may say that a minimal vector (5.2) has carrier $C_{n-R}$. By using the transformation (3.11), we see that the number of minimal vectors with carrier $C_{n-R}$ is the same for all cosets of dimension $n-R$; call this number $N_{R}$, so that

$$
2 s_{R}=2^{R} K_{n, R} N_{R} \text {. }
$$

For a minimal vector (5.2) with carrier a subgroup $V_{n-R}$, set

$$
x_{\boldsymbol{\alpha}}=2^{\lambda_{R}}\left(1-2 z_{\alpha}\right) \text { if } \alpha \epsilon V_{n-R}, \quad x_{\alpha}=0 \text { otherwise, }
$$

so that $z_{\boldsymbol{\alpha}}$ is defined on $V_{n-R}$ and has the value 0 or 1 . It may now be verified that $x \in \Lambda(\lambda)$ if and only if

$$
z \in \Lambda\left(0,0, \lambda_{R+2}-\lambda_{R}-1, \lambda_{R+3}-\lambda_{R}-1, \ldots, \lambda_{n}-\lambda_{R}-1\right)
$$

(a $2^{n-R}$-dimensional lattice), with each $z_{\alpha}=0$ or 1 .

We must now distinguish the cases: $\lambda_{R+2}=\lambda_{R}+2 ; \lambda_{R+2}=\lambda_{R}+1$.

(a) If $\lambda_{R+2}=\lambda_{R}+2,(5.5)$ implies that certainly

$$
z \in \Lambda(0,0,1,1, \ldots, 1), z_{\alpha}=0 \text { or } 1 \text {; }
$$

the number of solutions of (5.6) is precisely the number of solutions in $G F(2)$ of a set of equations of rank $\left(\begin{array}{c}n-R \\ 2\end{array}\right)+\ldots+\left(\begin{array}{l}n-R \\ n-R\end{array}\right)$, and nullity $1+\left(\begin{array}{c}n-R \\ 1\end{array}\right)$, i.e. it is $2^{1+n-R}$. Thus now

$$
N_{R} \leqq 2^{1+n-R}, 2 s_{R} \leqq 2^{n+1} K_{n, R} .
$$

This shows that the bound (5.1) is precise, i.e. that

$$
2 s_{R}=2^{n+1} K_{n, R} \text { if } \lambda_{R+2}=\lambda_{R}+2 .
$$

The same result is easily seen to hold if $\lambda_{R+2}$ is undefined, i.e. if $R \geqq n-1$.

(b) If $\lambda_{R+2}=\lambda_{R}+1$, then $\lambda_{R+3}=\lambda_{R+2}+1$ and (5.5) implies that

$$
z \in \Lambda(0,0,0,1,1, \ldots, 1), z_{\alpha}=0 \text { or } 1 \text {. }
$$

Arguing as above, we obtain

$$
N_{R} \leqq 2^{1+\left(\begin{array}{c}
n-R \\
1
\end{array}\right)+\left(\begin{array}{c}
n-R \\
2
\end{array}\right)},
$$

whence by $(5.3)$

$$
2 s_{R} \leqq 2^{n+1+\left({ }_{2}^{n-R}\right)} K_{n, R} .
$$

By a deeper investigation into the case $\lambda_{R+2}=\lambda_{R}+1$, based on the theory of $(n-R)$-dimensional quadratic forms over $G F(2)$, we have 
established the precise result

$$
2 s_{R}=2^{n+1} K_{n, R} \sum_{0 \leqq \delta \leqq d} 2^{\delta(\delta-1)} \frac{\left(2^{n-R}-1\right)\left(2^{n-R-1}-1\right) \ldots\left(2^{n-R-2 \delta+1}-1\right)}{\left(4^{\delta}-1\right)\left(4^{\delta-1}-1\right) \ldots(4-1)}
$$

where $d \geqq 0$ is the largest integer such that there are minimal vectors of ranks $R, R+2, R+4, \ldots, R+2 d$; alternatively expressed, $d$ is the largest integer for which

$$
\lambda_{R+i}=\lambda_{R}+\left[\frac{i+1}{2}\right] \text { for } 0 \leqq i \leqq 2 d .
$$

From (5.10), or pursuing the direct argument which led to (5.9), it follows that the bound (5.9) is precise if $(5.11)$ holds for all $i \geqq 0$. In particular, for the form $f_{N}$ whose exponents are given by (4.16) (or equivalently by (4.17)) we obtain

whence

$$
2 s_{R}=2^{n+1+\left({ }^{n-R}\right)} K_{n, R} \text { for all odd } R \leqq n,
$$

$$
s\left(f_{N}\right)=2^{n} \sum_{R \text { odd }} 2^{\left(\begin{array}{c}
n-R \\
2
\end{array}\right)} K_{n, R} .
$$

\section{Conclusion}

Our analysis of the lattices $\Lambda(\lambda)$ has yielded a large number of extreme forms $f_{(\lambda)}$, nearly all of which are new. The form $f_{(0, \ldots, 0,1)}$ is the known form $B_{N}$ of [2]. The special form $f_{N}$, corresponding to the exponent set $\lambda_{r}=\left[\frac{1}{2} r\right](0 \leqq r \leqq n)$, is equivalent to the known absolutely extreme form when $N=4$ or $N=8$, and may well be absolutely extreme for some larger $N$.

For all sufficiently large $N, f_{N}$ cannot be absolutely extreme, since $\gamma_{N}\left(f_{N}\right)$ is of order $N^{\frac{1}{2}}$ only. We can hope that, by methods similar to those used here, a sequence of forms can be constructed with larger values of $\gamma_{N}(f)$ for large $N$. A preliminary investigation suggests the existence of such a sequence with $\gamma_{N}(f)$ of order $N^{2 / 3}$ whenever $N=2.3^{n}$.

We add finally some notes on further results which may be obtained from our analysis of the lattices $\Lambda(\lambda)$.

(i) By choosing suitable sublattices of $\Lambda(\lambda)$ of lower dimension, it is possible to construct further forms with relatively large values of $\gamma_{N}(f)$. Thus the sublattice of $\Lambda(0,1,1,2)$ defined by

$$
\sum_{\alpha \in V_{3}} x_{\alpha}=0
$$

gives a 7 -variable form with $\gamma_{7}(f)=2^{6 / 7}$; since $\gamma_{7}=2^{6 / 7}$, this form is absolutely extreme. Similarly, the sublattice of $\Lambda(0,0,1,1,2)$ defined by

$$
\sum_{\alpha \in V_{4}} x_{\alpha}=0
$$


gives a 15 -variable form with $\gamma_{15}(f)=2^{7 / 5}$; thus we obtain the new inequalities

$$
\gamma_{15} \geqq 2^{7 / 5} ; \Delta_{15} \leqq 2^{1 / 0} .
$$

(ii) Many of our results will apply with very little modification to give upper bounds for the critical determinant of the $N$-dimensional convex body

$$
K_{v}: \sum_{\boldsymbol{\alpha} \in V}\left|x_{\boldsymbol{\alpha}}\right|^{\nu} \leqq 1 \quad\left(v \geqq 1, V=V_{n}\right) .
$$

For example, for $\boldsymbol{x} \in \Lambda(0,1,2, \ldots, n), \boldsymbol{x} \neq \mathbf{0}$, we find that

$$
\min \sum_{\boldsymbol{\alpha} \in V}\left|x_{\boldsymbol{\alpha}}\right|=2^{n}=N
$$

thus, for the 'octahedron' $K_{1}$, we have

$$
\Delta\left(K_{1}\right) \leqq d\left(N^{-1} \Lambda(0,1,2, \ldots, n)\right)=N^{-N} 2^{\mathcal{I r}\left(\begin{array}{l}
n \\
r
\end{array}\right)}=N^{-\frac{1}{2} N} \quad\left(N=2^{n}\right) .
$$

This represents an improvement on known results for small $N \geqq 4$.

We append a table of the distinct extreme forms $f_{(\lambda)}$ for $N=4,8,16$ and 32, giving the values of $\log _{2} M=m$; $\log _{2} D$; the number $s$ of pairs of minimal vectors; and $\log _{2} \Delta$ (where $\Delta=(2 / M)^{N} D$ ). The values of $\Delta$ for $f_{(0,0,1)}$ and $f_{(0,0,1,1)}$ show that they are the known absolutely extreme forms in 4 and 8 variables respectively. The italicized figures in the exponent sets $(\lambda)$ are the $\lambda_{R}$ for which there exist minimal vectors of rank $R$, i.e. for which $n-R+2 \lambda_{R}=m$.

\begin{tabular}{c|c|r|r|r|r}
\hline$N$ & $(\lambda)$ & $\log _{2} M$ & $\log _{2} D$ & $s$ & $\log _{2} A$ \\
\hline 4 & $(0,0,1)$ & 1 & 2 & 12 & 2 \\
8 & $(0,0,0,1)$ & 1 & 2 & 56 & 2 \\
& $(0,0,1,1)$ & 2 & 8 & 120 & 0 \\
16 & $(0,0,0,0,1)$ & & & & \\
& $(0,0,0,1,1)$ & 2 & 10 & 1,136 & -6 \\
& $(0,0,0,1,2)$ & 2 & 12 & 560 & -4 \\
& $(0,0,1,1,2)$ & 3 & 24 & 2,160 & -8 \\
& $(0,0,1,2,2)$ & 3 & 32 & 240 & 0 \\
32 & $(0,0,0,0,0,1)$ & & & & \\
& $(0,0,0,0,1,1)$ & 2 & 12 & 9,952 & -20 \\
& $(0,0,0,0,1,2)$ & 2 & 14 & 4,960 & -18 \\
& $(0,0,0,1,1,2)$ & 3 & 34 & 40,672 & -30 \\
& $(0,0,0,1,2,2)$ & 3 & 44 & 4,960 & -20 \\
& $(0,0,1,1,1,2)$ & 3 & 54 & 992 & -10 \\
& $(0,0,1,1,2,2)$ & 4 & 64 & 73,440 & -32 \\
& $(0,0,1,2,2,2)$ & 4 & 84 & 1,024 & -12 \\
& $(0,0,1,2,3,3)$ & 4 & 96 & 992 & 0 \\
& $(0,1,1,1,2,2)$ & 4 & 74 & 9,952 & -22 \\
\hline
\end{tabular}


We should like finally to express our thanks to Professor T. G. Room for helping us spot the transformation (4.12).

\section{References}

[1] Barnes, E. S., Criteria for extreme forms, This Journal p. 17.

[2] Coxeter, H. S. M., Extreme forms, Canad. J. Math. 3 (1951), 391-441.

[3] Koksma, J. F., Diophantische Approximationen (Springer, 1936).

The University of Sydney. 